\title{
Keine Gesundheitspolitik ohne die FMH
}

Eines ist klar: Die Abstimmung vom 1. Juni hat einen klaren Gewinner hervorgebracht - die Patientinnen und Patienten. Denn nie zuvor hat sich in der Schweiz eine derart breit gefächerte Allianz so vehement für deren Bedürfnisse eingesetzt. An der Seite von uns Ärztinnen und Ärzten kämpften in einem intensiven Abstimmungskampf Pflegende, Zahnärzte, Apotheker, Gesundheitsberufsverbände, Patienten- und Konsumentenorganisationen. Und dies überaus erfolgreich. Mit einem Nein-Anteil von 69,5 Prozent hat das Schweizer Stimmvolk dem Verfassungsartikel «Für Qualität und Wirtschaftlichkeit in der Krankenversicherung» eine wuchtige Absage erteilt. Dabei haben sämtliche Kantone die Verfassungsänderung abgelehnt, die Romandie noch stärker als die Deutschschweiz.

Das Resultat bedarf keiner Interpretation: Die Schweizerinnen und Schweizer wollen keine gesundheitspolitischen Experimente. Was sie wollen, ist ein ausgewogenes und qualitativ hochstehendes Gesundheitswesen, in dem verschiedene Krankenkassen agieren, wie wir seit der Abstimmung über die Einheitskasse wissen - und in dem die Position dieser Kassen klar definiert ist, wie wir nun seit dem 1. Juni wissen.

Dass dazu auch Reformen notwendig sind, ist unbestritten. Eine zentrale Rolle bei der Gestaltung dieser Reformen wird in Zukunft genau dieser Allianz zufallen, die den Abstimmungskampf geführt hat. Die neu gewonnene Rolle als Keyplayer muss nun genutzt werden, um das schweizerische Gesundheitswesen im Sinne der Patientinnen und Patienten weiterzugestalten.

Die Regelung der Gesundheitsversorgung durch die öffentliche Hand, aber auch die freie Arztwahl oder die Übernahme der spitalexternen Pflegekosten gehören mit Sicherheit dazu. Dabei sind - wie das Stimmvolk richtig erkannt hat - verschiedene Kompetenzebenen zu beachten.
Aufgabe der öffentlichen Hand ist es, die Rahmenbedingungen des Gesundheitswesens zu definieren und es zu regeln - zum Beispiel den Zugang zur Pflege oder die medizinische Demographie. Diese Aufgabe kommt keiner anderen Instanz zu, schon gar nicht den Versicherern. Dass die Ärzteschaft dabei in die verschiedenen Entscheidungsprozesse eingebunden werden muss, steht nicht zur Diskussion.

Innerhalb der politisch definierten Rahmenbedingungen müssen dann Leistungserbringer und Versicherer gemeinsam sicherstellen, dass das Gesundheitswesen funktioniert - Stichworte sind hier Tarife, Qualität, Rechnungskontrollen usw.

Diese beiden Kompetenzebenen dürfen nicht mehr verwechselt werden.

Nach dem 1. Juni geht es nun darum, die durch den Abstimmungskampf gewonnene Dynamik zu nutzen, um Lösungen zu erarbeiten, Projekte voranzutreiben und Anträge durchzubringen. Die Stimme der Ärzteschaft wird dabei besonderes Gewicht haben, denn die FMH konnte ihre Rolle als wichtige Leaderin in der Gesundheitspolitik nachhaltig festigen - notabene als eine Leaderin, die Hand zu Modellen bietet, die sich in der Praxis bewähren.

Ende Monat präsentiert die FMH der ständerätlichen Kommission für soziale Sicherheit und Gesundheit ihre Lösungsvorschläge in bezug auf die Nachfolgeregelung zum Zulassungstopp und in bezug auf die Steuerung des Gesundheitswesens. Diese basieren unter anderem auf Modellen, die in verschiedenen Kantonen bereits erfolgreich angewendet werden und die sich schweizweit adaptieren und übernehmen lassen, aber auch auf Grundlagen, welche die FMH in den letzten Monaten erarbeitet hat.

Dr. med. Jacques de Haller Präsident der FMH 